\title{
Step Ladder Expansive Cranioplasty: Feasibility Study on Computed Tomographic Scan Images
}

\author{
S. K. Sengupta ${ }^{1}$ Hirdesh Sahni $^{2}$ T. J. Rappai ${ }^{3}$ Saurabh Verma ${ }^{4}$ \\ ${ }^{1}$ Department of Neurosurgery (Surgical Ward III), Base Hospital Delhi \\ Cantonment, New Delhi, India \\ 2 Department of Radiology and Intervention radiology, Base Hospital \\ Delhi Cantonment, New Delhi, India \\ ${ }^{3}$ Department of Neurosurgery, Command Hospital Eastern \\ Command, Kolkata, India \\ ${ }^{4}$ Department of Neurosurgery, Command Hospital Southern \\ Command, Pune, Maharashtra, India
}

Indian J Neurosurg 2015;4:102-108.

\begin{abstract}
Address for correspondence Col (Dr.) S. K. Sengupta, MS (Gen Surg), MCh (Neurosurgery), Department of Neurosurgery (Surgical Ward III), Base Hospital Delhi Cantonment, New Delhi 110010, India (e-mail: sudipkumarsengupta14@rediffmail.com).
\end{abstract}

\begin{abstract}
Background A novel technique of step ladder expansive cranioplasty was suggested, in a recent study, as a credible alternative to decompressive hemicraniectomy and found feasible on evaluation on a mathematical model, where human cranium was represented by a hemisphere of $9 \mathrm{~cm}$ radius. The findings on the mathematical model required to be validated in clinical setting before planning any clinical trial.

Methods A retrospective analysis of cases of decompressive craniectomy performed at a single neurosurgery center over past 18 months was done. Cases in which an NCCT head was repeated on the first postoperative day, due to evidence of intractable raised ICP postoperatively, were included in the study. The pre- and postoperative films were evaluated to determine various parameters, namely the thickness of skull bone at the craniectomy margins, projection of dural outpouching beyond the craniectomy defect, and the height required to be gained by an expansive cranioplasty to accommodate the augmented volume of the dural sac.

Results Six cases that satisfied all criteria were included. The mean surface area of craniectomy defects were $66.89 \mathrm{~cm}^{2}$ in size. The maximum projection of the dural

\section{Keywords}

- decompressive craniotomy

- step ladder expansive cranioplasty

- NCCT images outpouching, as measured from the craniectomy margin, was $2.7 \mathrm{~cm}$, necessitating construction of an expansive cranioplasty achieving $1.6 \mathrm{~cm}$ of gain in height in the cranium with minimum calvarial convexity.

Conclusion The results of this study indicate that a two-step expansive cranioplasty can accommodate adequate volume expansion in individuals whose average calvarial thickness at the craniectomy defect is $0.8 \mathrm{~cm}$.
\end{abstract}

\section{Introduction}

Ability of decompressive craniectomy to reduce intracranial pressure is well established. ${ }^{1-3}$ However, it comes at the cost of second surgery in the form of cranioplasty and certain complications in the form hydrocephalus and

received

April 30, 2015

accepted

May 11, 2015

published online

July 29, 2015 "syndrome of trephined." ${ }^{4}$ Various novel surgical techniques have been suggested, claiming varied success, with an aim to be able to put the bone flap back in the same sitting. ${ }^{5,6}$ Recently a concept of an expansive cranioplasty was proposed as a credible alternative, based on a mathematical model. ${ }^{7}$
License terms of India 
In the mathematical model, it was presumed that dura forms an 18-cm-diameter hemispherical watertight bag containing brain floating in cerebrospinal fluid (CSF), with the flat circular surface of the hemisphere lying over the base of the skull. Human cranium, however, is not symmetrical and has great variation in size and shape. The findings on the mathematical model required to be validated in clinical setting before planning any clinical trial.

\section{Methods}

A retrospective analysis of a prospectively collected data was done. All cases of decompressive craniectomy performed at a single neurosurgery center over past 3 years were screened. In absence of facility for intracranial pressure (ICP) monitoring at the single neurosurgery center, the decision to take up a case for decompressive craniectomy was based on clinical suspicion of raised ICP, lateralizing signs, and computed tomographic (CT) scan of the head showing obliteration of basal cisterns, and/or significant midline shift. In all but five cases an expansive duraplasty was done. Craniectomy defects were oval in every case with anteroposterior diameter being the largest.

Cases in which a noncontrast CT (NCCT) of the head was repeated on the first postoperative day, due to evidence of intractable raised ICP postoperatively, were included in the study.

Exclusion criteria are listed as follows:

1. Cases where a CT scan was repeated on clinical suspicion of an intractable raised ICP, but the neuroimaging showed preserved basal cisterns and near resolution of midline shift.

2. Cases where dural closure was not done.

3. Cases that had undergone significant internal decompression along with the decompressive hemicraniectomy.
NCCT images of the head were acquired in a 16-slice spiral CT machine. Images were saved on CD and transferred on another device where various measurements were done using OSIRIX software.

The pre- and postoperative CT scans were opened on two parallel windows on OSIRIX and ensured that they were sections obtained at planes equidistant from a fixed bony landmark (tip of the left clinoid process) in the base of the skull.

Craniectomy defects were measured at the sections where they had maximum diameter, at the level of the inner table. Thickness of the cranium at the site of the craniectomy defect was measured. The distance of the dural margin from the craniectomy defect was measured at the point where it was maximum by dropping a perpendicular line to the line connecting the craniectomy edges at the level of the inner table. All measurements were repeated in both axial and coronal plains (-Fig. 1).

On the axial cut images, the distance of the craniectomy margin at the anterior end was measured from a fixed bony point in the midline. A similar line of equal length (correct to the second decimal point in millimeter) from the same bony point was drawn on the preoperative image. From the lateral most point of this line, another line was drawn of the length equal to that of the craniectomy defect anteroposteriorly. A perpendicular was drawn from the midpoint of this line to the inner cortex and its length recorded (-Fig. 2). Similar measurement was also taken at a point, other than the midpoint, where the distance between this imaginary line and the inner cortex was maximum. The smaller of the two measurements was recorded and used for the study. Measurements were repeated in the coronal sections with the midline being the standard of reference (-Fig. 3).

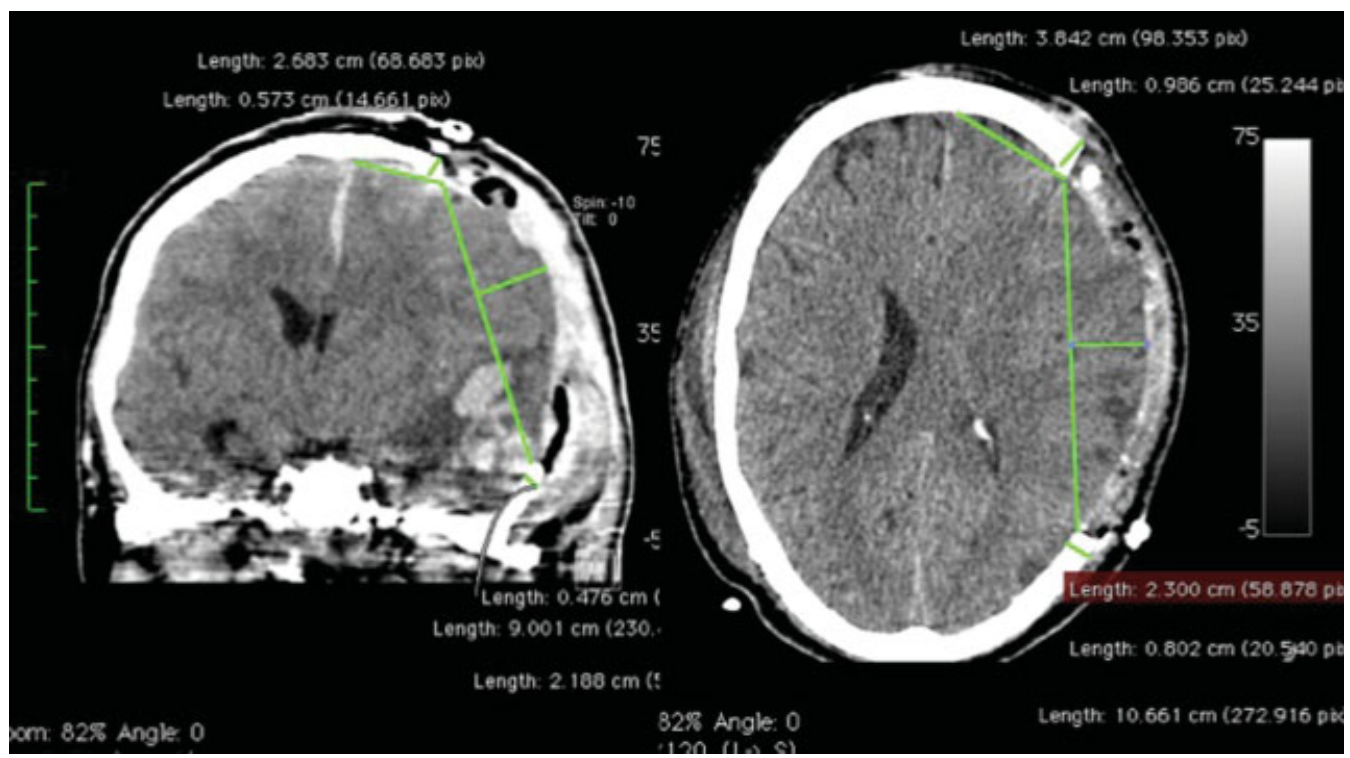

Fig. 1 Postoperative noncontrast computed tomographic images in coronal and axial sections in a case of traumatic brain injury. Size of the craniectomy defect has been measured by drawing a straight line at the level of the inner table at the edge of the defect. A perpendicular line has been drawn from the previous straight line to the outer margin of the dura at the point of maximum width. Thickness of the cranium at the site of the craniectomy defect was measured in axial section. 

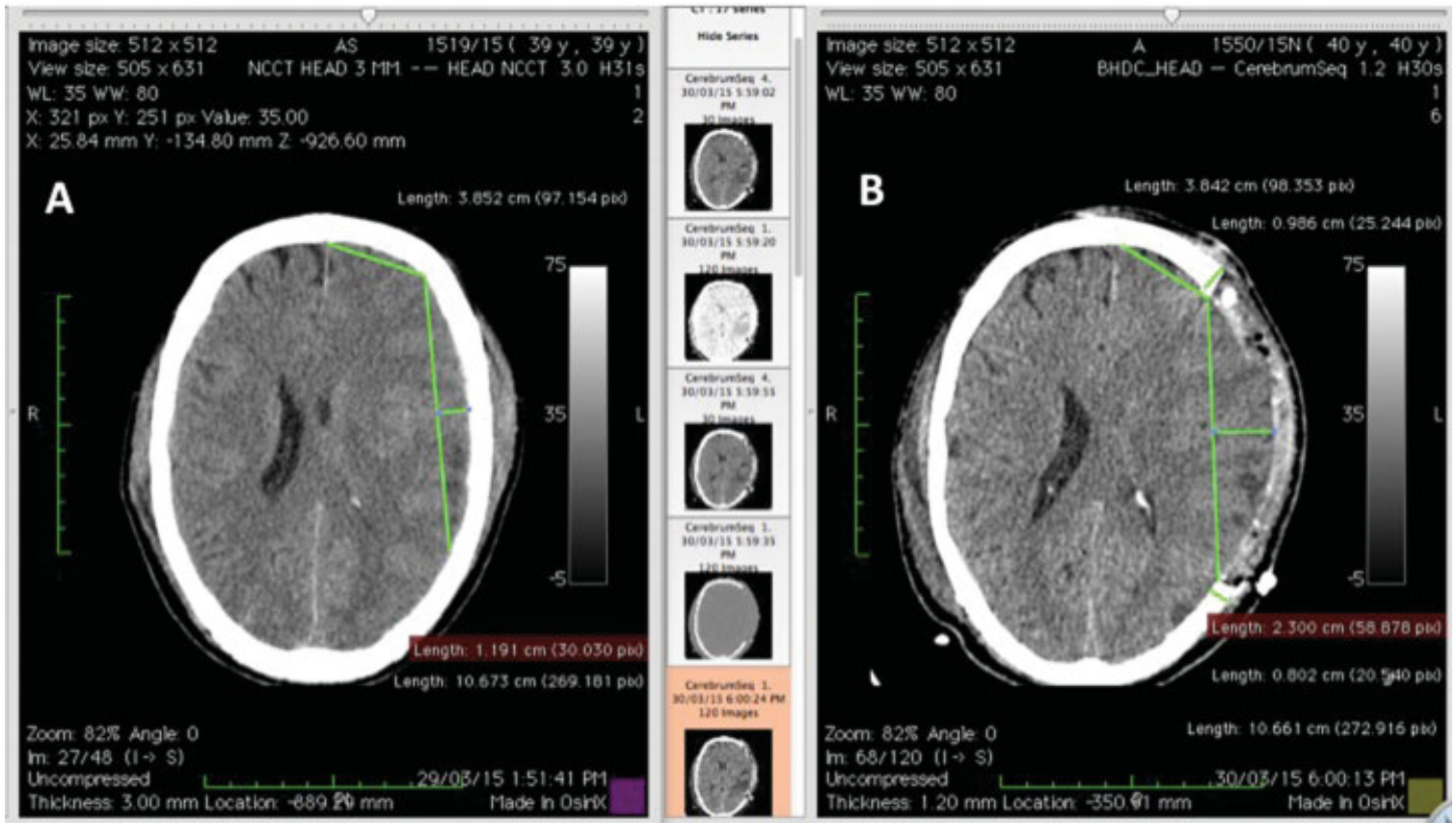

Fig. 2 (A, B) Pre- and postoperative axial sections of each case were displayed on two adjacent windows. The distance of the craniectomy margin at the anterior end was measured from a fixed bony point in the midline. A similar line of equal length from the same bony point was drawn on the preoperative image. From the lateral most point of this line, another line was drawn of the length equal to that of the craniectomy defect anteroposteriorly. A perpendicular was drawn from the midpoint of this line to the inner cortex and its length recorded.

The pre- and postoperative films were evaluated to determine following parameters:

1. Thickness of skull bone at the craniectomy margins

2. Maximum outward projection of dural outpouching beyond the craniectomy defect in axial and coronal plane
3. The distance of the inner table in the preoperative skull from the imaginary plane of craniectomy, indicating the preoperative position of the dural bag

4. The height required to be gained by an expansive cranioplasty to accommodate the augmented volume of the dural sac
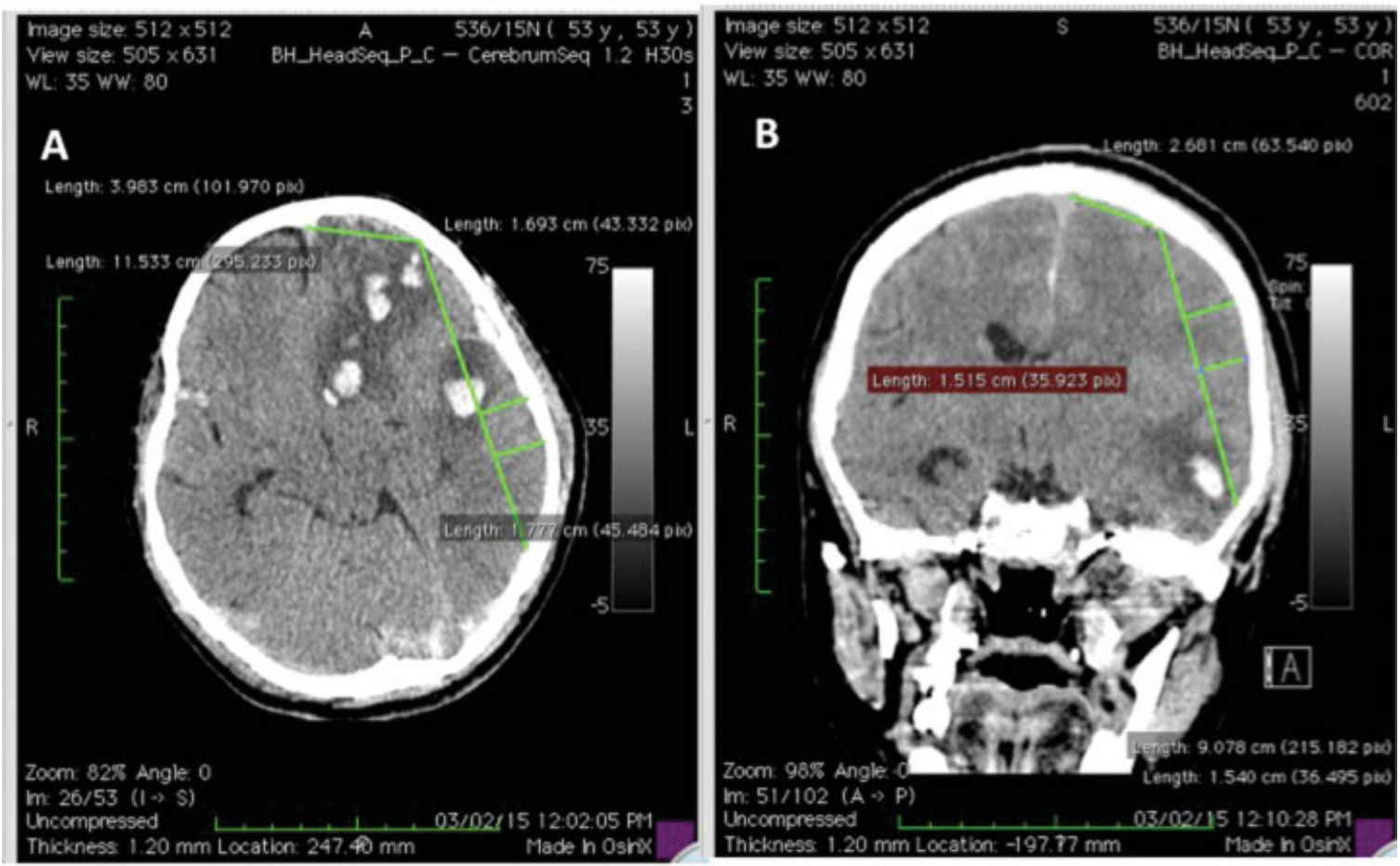

Fig. 3 (A, B) Axial and coronal preoperative CT scans of a case of severe head injury showing measurement of preoperative dural margins. 


\section{Results}

A total of 31cases of decompressive hemicraniectomy were performed at our center from October 1, 2013, to March 31, 2015. In all but five cases, expansive duraplasty was done. Indication for performing a decompressive hemicraniectomy was malignant middle cerebral artery (MCA) infarct in seven cases, hypertensive intracerebral bleed in two cases, and rest were cases of traumatic brain injury. In eight cases, there was clinical suspicion of unresolved intractable raised intracranial pressure postoperatively necessitating a repeat neuroimaging. However, two patients died before any neuroimaging could be arranged. One patent was transferred on ventilator to a center of choice of his next of kin. Six patients underwent an NCCT head within first 24 hours of surgery to rule out the requirement of any further neurosurgical intervention. One case where a CT scan was repeated on clinical suspicion of an intractable raised ICP, but the neuroimaging showed preserved basal cisterns and near resolution of midline shift, was excluded. In one case of severe head injury with acute subdural hematoma (SDH) in which, after evacuation of SDH bone flap was replaced, developed cerebral edema, clinical evidence of raised ICP with lateralizing sign, and showed significant midline shift on NCCT of the head, necessitating removal of the bone flap on the third postoperative day. This case has been included in the study. The results have been summarized in - Table 1.

The mean surface area of craniectomy defects was 66.89 $\mathrm{cm}^{2}$ in size (max $81.32 \mathrm{~cm}^{2}$ and $\min 54.22 \mathrm{~cm}^{2}$ ). The maximum projection of the dural outpouching, as measured from the craniectomy margin, was $2.7 \mathrm{~cm}$ (mean $2.25 \mathrm{~cm}$, mode $2.4 \mathrm{~cm}$ ). Projection of the dural outpouching was more on the axial plane in each case (mean $2.33 \mathrm{~cm}$ ) as compared with that on the coronal plane (mean $2.11 \mathrm{~cm}$ ), and unlike in axial plane where in four out of five (80\%) cases the maximum outpouching was over the midpoint of the imaginary line joining the craniectomy edges, only in one out of five (20\%) cases the maximum outpouching was over the midpoint in coronal plane. In one case where there was a significant postoperative epidural hematoma (EDH), the lateral most limit of the EDH and the dural pouch were found equidistant from the plane of the craniectomy defect. The distance of the inner table in the preoperative skull from the imaginary plane of craniectomy, indicating the preoperative position of the dural bag was found to be greater in each case on the axial plane (mean $1.46 \mathrm{~cm}$ ) as compared with that in the coronal plane (mean $1.36 \mathrm{~cm}$ ) and except in one case (case 5 in -Table 1), the distance increased with increase in craniectomy surface area in all cases.

\section{Discussion}

The fact that decompressive craniectomy effectively reduces intracranial pressure is well established. ${ }^{1-3}$ Various novel surgical techniques have been suggested, claiming varied success, with an aim to be able to put the bone flap back in the same sitting. ${ }^{5,6}$ However, they have been conducted in very small number of cases, and none have measured the ICP reduction or volume expansion achieved .The concern that leaving the bone flaps back under the scalp flap will restrict the available space and will put additional pressure on the brain parenchyma has not been addressed in any of these studies. This has resulted in lack of acceptance of these techniques in general practice.

Recently a concept of an expansive cranioplasty was proposed as a credible alternative, based on a mathematical model. ${ }^{7}$ In the mathematical model it was presumed that dura forms a 9-cm-radius hemispherical watertight bag containing brain floating in CSF, with the flat circular surface of the hemisphere lying over the base of the skull (-Fig. 4). It was also presumed that the outpouching of the dural bag, after a decompressive hemicraniectomy and expansive duraplasty, can be represented by a section of an imaginary sphere. Mathematical calculations on the model showed that a projection of dural outpouching of $1.32 \mathrm{~cm}$ beyond the craniectomy margin of a unilateral $12 \times 15 \mathrm{~cm}$ elliptical craniectomy defect is required, to achieve and accommodate a volume expansion of $124 \mathrm{~cm}^{3}$, which was recorded to be the mean volume expansion in the study published by Olivecrona et al, ${ }^{8}$ reporting a positive outcome of the study.

Human cranium, however, is not symmetrical and has great variation in size and shape. The findings on the mathematical model required to be validated in clinical setting before planning any clinical trial. This necessity was the reason behind the genesis of the current paper.

At our center, ICP monitoring is not undertaken routinely and the decision to take up a case for decompressive craniectomy is based on clinical suspicion of raised ICP, lateralizing signs and CT scan of the head showing obliteration of basal cisterns and/or significant midline shift. Neuroimaging is repeated in postoperative cases only when there is clinical suspicion of resistant raised ICP postoperatively or there is suspicion of hydrocephalus.

\section{Justification for Setting the Inclusion Criteria}

Volume augmentation is directly proportional to the craniectomy size. ${ }^{9}$ It is also dependent on the difference between the intracranial pressure pushing the scalp flap outward and the tensile strength of the scalp flap along with the atmospheric pressure resisting any expansion. Hence the volume expansion recorded in the same individual will be different at different levels of ICP. Because this study aimed to find out the maximum volume augmentation required to be accommodated by expansive cranioplasty, selecting only those cases that had clinical and radiological evidence of raised ICP at the time of neuroimaging was aimed at taking care of this bias.

\section{Justification for Setting the Exclusion Criteria}

1. Cases where post-op CT scan showed preserved basal cisterns and near resolution of midline shift: In absence of availability of ICP recordings, these CT findings were considered indications of normal ICP. 


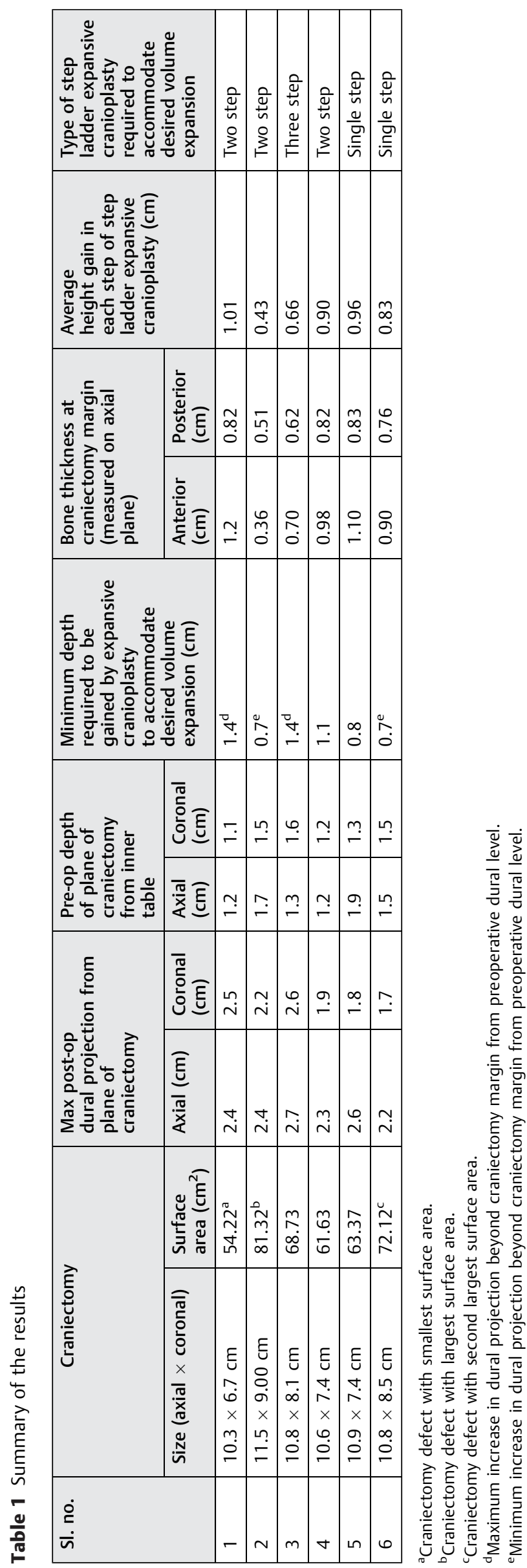

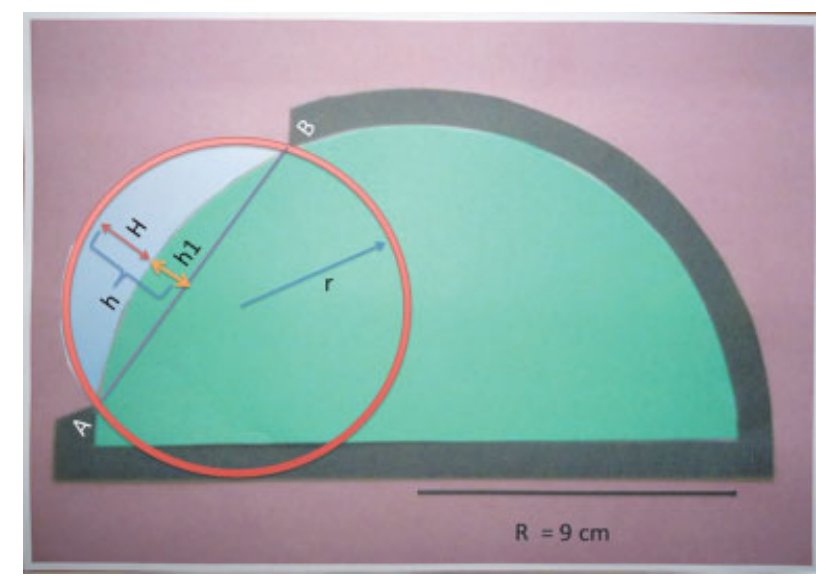

Fig. 4 Mathematical model: Black border represents the skull and its discontinuity represents craniectomy defect. Green area represents the hemispherical intracranial dural bag in the preoperative skull with radius $R$. For a hemisphere of $1,500 \mathrm{cc}$ volume $R$ works out to be approximately $9 \mathrm{~cm}$. A line $A B$ joining the inner table at the level of the craniectomy defect represents the plane of the craniectomy defect. $h 1$ represents the maximum distance of the inner table the level of craniectomy defect in preoperative skull. After volume augmentation of the dural sac (represented by blue area), forms a part of an imaginary circle of radius $r$ represented by the red circle. At the craniectomy site the maximum projection of the dural sac beyond the craniectomy margin $h$ is the sum of preoperative height (as measured from craniectomy plane), that is, $h 1$ and the postoperative increment $H$.

2. Cases where dural closure was not done: In cases where a dural closure has been achieved, the shape of a dural outpouching is likely to represent a cut portion of a sphere (7). However, when dura has not been closed, the resulting brain herniation can assume any asymmetrical shape. For any given volume the height of the dural outpouching from the craniectomy margin would be maximum for a spherical projection. Because this study aimed to find out the feasibility of an expansive cranioplasty that can accommodate the outward expansion of the dural bag, cases where dural closure was not done were excluded.

Our present study results closely replicated the findings of study done on the mathematical model ${ }^{7}$ and the study by Cavuşoğlu et al. ${ }^{10}$ However, the height of the dural outpouching from the craniectomy margin increases proportional to the size of the craniectomy. ${ }^{7,10}$ It was proven on the mathematical model that for a given volume expansion the projection of dural outpouching beyond the preoperative dural limit is less for a larger craniectomy size.

The proposed technique of step ladder expansive cranioplasty ${ }^{7}$ consists of cutting out concentric bony rings from the free bone flap and fixing the flaps on the opposite surfaces of the miniplates before placing them back to construct the expansive cranioplasty (-Fig. 5).

A two-step expansive cranioplasty could have been constructed to accommodate a postcraniotomy augmentation of dural outpouching of $1.4 \mathrm{~cm}$ from the 


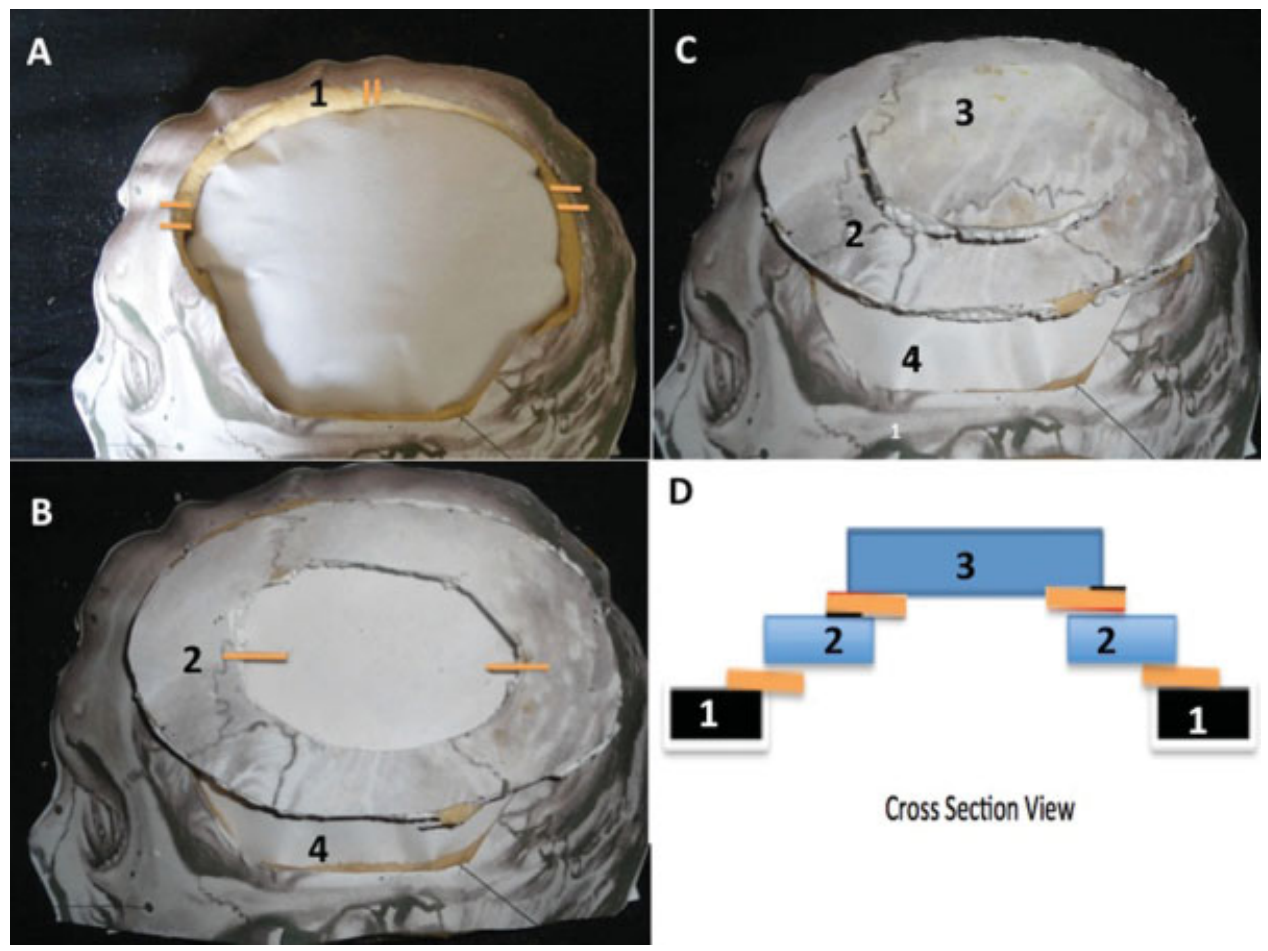

Fig. 5 Step ladder expansive cranioplasty: (A) Model showing craniectomy defect 1. (B) The free bone flap has been cut in two concentric rings. The outer ring 2 has been fixed by fixing the miniplates to the inner table of the bone flap. (C) A completed two-step step ladder expansive cranioplasty constructed by fixing the free bone flap and the cranium on the two opposite surfaces of a titanium miniplate. 3 represents the inner ring carved out of the free bone flap, and 4 represents portion of dura over the temporal region left without a bony cover (D) Crosssection of a two-step step ladder expansive cranioplasty. Yellow lines represent titanium miniplates.

craniectomy margin, the maximum in the present study in any individual with average skull bone thickness of $0.7 \mathrm{~cm}$, which was true for four out of six patients in the study.

It can be argued that the cases included in this study obviously had undergone inadequate decompression, since they continued to have refractory raised ICP, hence topping them up with an expansive cranioplasty would have only added to the insult. On the other hand, whatever was the extent of surgery planned by the surgeon was already done and the volume augmentation already achieved, an expansive cranioplasty that can take the inner table way beyond the margin of the dura could not have caused any further damage, on the contrary by taking off the inward force exerted by the tensile strength of the scalp and the atmospheric pressure it could have helped in reducing the ICP further.

The surgical nuances can be worked out to finer details before commencing a clinical trial especially with a view to achieving a satisfactory bony union.

The present study was done on patients in whom an expansive cranioplasty was not planned preoperatively. Patients can be chosen for this procedure, based on their skull bone thickness calculated on the preoperative neuroimages. If an expansive cranioplasty is in the mind of the surgeon, the craniotomy defect can be suitably sited to our advantage. Two additional measures to improve the volume augmentation will be to leave the temporal part of the craniectomy defect without any bony cover and create a little extra space by drilling out a circular area of inner table and dipole from the center of the bone flap. Leaving a craniectomy defect of $3 \mathrm{~cm}$ diameter at the center of the bone flap can also be considered as an alternative.

\section{Limitations of the Study}

1. Only six cases were included in the study.

2. Axial images were acquired by the CT scanner while coronal images were created by volume rendering by the computer software accepting any distortion in threedimensional (3D) representations, and in measurements resulting from the process.

3. Absence of facility for ICP measurement.

\section{Conclusion}

This study results indicate that a two-step expansive cranioplasty can accommodate adequate volume expansion while alleviating the ill effects of a craniectomy and necessity of a second surgery in individuals whose average calvarial thickness at the craniectomy defect is greater than $7 \mathrm{~mm}$. This novel surgical technique can be tried in clinical setting in selected individuals with adequate calvarial thickness determined by the preoperative NCCT. The surgical nuances need to be worked out to finer details before commencing a clinical trial especially with a view to achieving a satisfactory bony union. 
108 Step Ladder Expansive Cranioplasty: Feasibility Study Sengupta et al.

\section{Disclosure \\ None to declare. \\ Conflict of Interest \\ None to declare.}

\section{References}

1 Jiang JY, Xu W, Li WP, et al. Efficacy of standard trauma craniectomy for refractory intracranial hypertension with severe traumatic brain injury: a multicenter, prospective, randomized controlled study. J Neurotrauma 2005;22(6): 623-628

2 Bao YH, Liang YM, Gao GY, Pan YH, Luo QZ, Jiang JY. Bilateral decompressive craniectomy for patients with malignant diffuse brain swelling after severe traumatic brain injury: a 37-case study. J Neurotrauma 2010;27(2):341-347

3 Yoo DS, Kim DS, Cho KS, Huh PW, Park CK, Kang JK. Ventricular pressure monitoring during bilateral decompression with dural expansion. J Neurosurg 1999;91(6):953-959

4 Yamaura A, Makino H. Neurological deficits in the presence of the sinking skin flap following decompressive craniectomy. Neurol Med Chir (Tokyo) 1977;17(1, Pt 1):43-53
5 Valença MM, Martins C, da Silva JC, et al. An innovative technique of decompressive craniectomy for acute ischemic stroke. In: Balestrino M, ed. Advances in the Treatment of Ischemic Stroke. InTech; 2012. Available at: http://www.intechopen.com/books/ advances-in-the-treatment-of-ischemicstroke/decompressivecraniectomy-for-cerebral-ischemia

6 Peethambaran AK, Valsalmony J. Four-quadrant osteoplastic decompressive craniotomy: a novel technique for decompressive craniectomy avoiding revision cranioplasty after surgery. Neurol India 2012;60(6):672-674

7 Sengupta SK. Step-ladder expansive cranioplasty after decompressive craniotomy and expansive duraplasty: discussing possibilities on a mathematical model. Indian J Neurosurg 2015;4:15-21

8 Olivecrona M, Rodling-Wahlström M, Naredi S, Koskinen LO. Effective ICP reduction by decompressive craniectomy in patients with severe traumatic brain injury treated by an ICP-targeted therapy. J Neurotrauma 2007;24(6):927-935

9 Skoglund TS, Eriksson-Ritzén C, Jensen C, Rydenhag B. Aspects on decompressive craniectomy in patients with traumatic head injuries. J Neurotrauma 2006;23(10):1502-1509

10 Cavuşoğlu H, Kaya RA, Türkmenoğlu ON, Aydin Y. Value of early unilateral decompressive craniectomy in patients with severe traumatic brain injury. Ulus Travma Acil Cerrahi Derg 2010; 16(2):119-124 